\title{
Pharmaceuticals and personal care products in a drinking water resource of Yangtze River Delta Ecology and Greenery Integration Development Demonstration Zone in China: occurrence and human health risk assessment
}

\author{
YANPING DUAN $^{1}$ \\ ${ }^{1}$ School of Environmental and Geographical Sciences, \\ Shanghai Normal University,100 Guilin Road, Shanghai, \\ 200234, China
}

The occurrence, partition, and human health risk of thirteen pharmaceuticals and personal care products (PPCPs) have been investigated in surface water, overlying water, pore water and sediment samples from Dianshan Lake of Yangtze River Delta Ecology and Greenery Integration Development Demonstration Zone in China. PPCPs were ubiquitous in aqueous phase and sediments from Dianshan Lake. Sulfamethazine was dominated in surface water and overlying water, while ketoprofen was rich in sediment. The total concentration of PPCPs ranged from 0.38-85.27 ng/L, 24.26-130.04 $\mathrm{ng} / \mathrm{L}$ and $5.39-149.84 \mu \mathrm{g} / \mathrm{kg}$ in surface water, overlying water and sediment, respectively, which were in middle levels compared with these reported in other aquatic environment in China. Naproxen, sulfadimethoxine, sulfamethoxazole and sulfamethazine in surface water showed a relatively higher level in lake side than those in lake center suggesting that a mixed containment source of human- and animal-derived from the areas around lake. The significant season variations of most PPCPs were mainly attributed to their usage, water temperature and dilution effect. The partition behaviors of PPCPs in sediment-overlying water and sediment-pore water system were mainly affected by their $\log$ Kow values, and showed weak correlation with total organic carbon content in sediment and molecular weights of PPCPs. Preliminary results indicated that PPCPs in Dianshan Lake have not posed a high risk to human health by exposure to drinking water for all age groups. Nevertheless, their potential to cause the mixture toxicity and resistance genes cannot be neglected. This work will contribute to the clear picture of PPCPs contamination in drinking water source in the Demonstration Zone, and provide reliable and simple-to-use information to regulators on the exposure and risk levels of PPCPs, as well as recommendations for future research. 